\title{
Ludic Engagement Designs: Creating Spaces for Playful Learning
}

\author{
Eva Petersson Brooks \\ Centre for Design, Learning and Innovation, Department for Architecture and \\ Media Technology, Aalborg University Esbjerg, Niels Bohrs Vej 8, \\ 6700 Esbjerg, Denmark \\ ep@create.aau.dk
}

\begin{abstract}
The learning within and the design of a learning (or therapeutic) situation constitutes a situated activity with inherent actions and interventions. The participant profile influences the facilitator's decisions on how to set up the attributes of the environment relative to the desired learning process and the expected outcome of that process. This paper presents a model which was developed relative to the development, use and evaluation of interactive spaces for playful learning. However, the model has a more generic value as it has been used in learning situations where other forms of resources and/or methods have been used. Thus, the general results upon which the present model is created, indicate that a playful learning tool may be construed by an open-ended design, in the sense that its (im)material affordances should in a flexible way support inclusion of different forms of emergent interaction and forms of play.
\end{abstract}

Keywords: playful learning, design, semiotic interplay, facilitation.

\section{Introduction}

This contribution introduces the concept of 'Ludic Engagement Designs' $[1,2]$. The body of work includes an emerged model for design and intervention that transcend and cross-inform in learning and rehabilitation situations. The term 'Ludic' relates to the designed for fun/playful participant-experience for both participant (learner) and facilitator (teacher/healthcare professional). 'Engagement' refers to the targeted immersion of the participant that is achieved through the adaptation of the available environment design features so that personalisation is optimised and, thereby, creating attention and consistency through agency and by means of navigation; a semiotic interplay. In other words, ludic engagement designs include the creation of spaces for playful learning.

The paper discusses affordances of interactive environments targeting learning and rehabilitation situations as well as the participants' playful engagements and inherent semiotic interplay. In doing so, the author will use examples from a wide range of empirical material (observations, video recordings, interviews) where interactive environments have been used for learning and training in the contexts of schools, museums and rehabilitation. The participants are children and young people; typically 
developed and with special needs. The methods used were based on a participatory design approach.

\section{Ludic Engagment Designs}

It has been shown in previous research that explorations are involved in the mastery of tools and in the growth of competence $[3,4,5,6]$. In the study presented in [6], the children (with severe special needs) were dynamically exploring what was occurring as a result of their empowered control. Their discovery of the interactive space involved range, speed, and directional variation of their gestures. Children especially indicated an early awareness, observed as improved concentration, which was a result of the direct correspondence and control to the physical movement of the robotic head and the subsequent movement of the light. Such self-achievement is a rare commodity for these children. This is relative to [3] where the absence of negative consequences encouraged the participants' exploration, which resulted in development of unemployed skills. This can also be applied to the parallel findings in [5] where the children with social and behavioral disabilities felt so safe and relaxed so that, for example, they closed their eyes in front of everybody when they were asked to do so, without any questioning or unwillingness. They were engaged in the interactive situation without expectations or rules, which resulted in creative achievements through exploration and expression. Through exploring the interactive environment the participant could acquire new abilities, interactions, expressions and emotions, enabling a mastering of tasks and practicing of skills. As such, explorative actions in the interactive environment created action cycles that had the potential to evoke the child's interest in practicing otherwise limited skills, which is vital in facilitation of achieving an optimal experience. Thus, I would like to suggest, that through practicing of skills the participant experienced a sense of control and, thereby, mastery and consciousness of the therapy situation and beyond.

\subsection{Transformation of Actions}

As described in [4] the initial patterns of actions became the constituents for new patterns of action through exercise. [7] extends this theory by emphasizing that the introduction of mediating tools created an imbalance in the systemic organization of the actions, which enhanced changed action patterns. Similarly, findings from the studies presented in $[5,6,8,9]$ demonstrated individual variation in action cycles. For example, one of the participant in [5] showed through his facial expressions that he went through different phases of interaction where intense concentration was balanced by relaxation, yet all aspects of the physical movements were intentional within his limits. The early sessions with a participant in [8] had instances of significance where his actions signaled his desire to participate more fully with increasingly extended instances of hand gestures. The participants involved in the study presented in [6] varied the range, the speed, and the direction of their gestures. The spatial movements by the participants in the study presented in [9] demonstrated variations in 
actions through range, intentionality, and shift of gestures. the support from the system encouraged the participants to reach beyond their limits; thus, achieving an extenuation of the participant's potentials. Thereby, I suggest that the transformation became apparent through the form of creative actions. I see a differentiation between creative and routine actions, where the creative actions are connected to the possible and the routine actions are connected to the existing. The functions of creative actions are the generation and creation of something new. Creative actions are not only characterized by motives, goals, and operations, but also by acts that generate new motives, goals, or operations [10]. By facilitating a safe environment creative actions are enhanced within the interactive space as an important prerequisite for playful learning processes. By this, I see action cycles as precondition for playful engagement.

\subsection{Traits of Playful Learning}

In his writings [11] has pointed to play as a source of the child's development through the Zone of Proximal Development (ZPD) which is defined by the distance between the child's actual level of development and the potential level of development. [12] characterizes play as a precondition to flow, which means an integration of physical, emotional, and mental functions. This was supported by the play activities as described in [5, 6]. As emphasized by [13] exploration goes hand in hand with play, but is not the same as play, rather explorations serve as a precondition to play. This is exemplified by the findings presented in [5] where the system offered exploratory and expressive elements, which was defined as an activity in which the participants were engaged in for fun and for their own sake; just like play situations - without expectations or rules. [14] underlines that interest has a motivating character that channels the child's choices involved in 'doings'. By this, the interest had an immediate character, which placed the participant in the midst of the play experience; that is to say that the participants became absorbed by the 'here and now', as in a flow state. As pointed to in [5] the findings demonstrated play in the form of creative achievements originating from explorations and expressive elements that motivated the sense of flow. As such the interactive play situations indicated an enhancement of the quality of play and learning, which, in turn, facilitated engaging explorations that were utilized in the therapy. This is to say that play had a motivational potential achieved from the interactive environment, which was apparent through the participant's concentration when interacting within the environment.

Findings from the participants' exploration and play within the interactive environment pointed towards awareness and enjoyment as the interaction empowered them to manipulate the technical device. The physical relationship of synchronized participant movement within the interactive space reinforced the participants' actions. This finding was supported by the studies presented in [8, 9]. I argue that this reinforcement of actions is directed by the participant or by the facilitator's concern with overcoming of restrictions and with the development of new and improved actions. In the study presented in [9] findings showed that the participants' use of the body when navigating within the computer game environment as well as the immediate feedback in the form of game content had the potential to reduce the physical load in the daily 
physical rehabilitation. Findings reported in [6] emphasize the immediate feedback that supported the participant to incrementally push their movement limits. All in all, these results point to playful learning potentials from actions and interactions within interactive environments. I consider play as a crucial foundation for playful learning and it was evident in the findings that the interactive play was closely connected to learning aspects exemplified mainly through the effects of the participant's feedforward (movements) resulting in self achievement, which is commonly rare for individuals with severe disabilities. The findings pointed to the components of (i) the merging of action and awareness, (ii) concentration, and (iii) the melting together of actions and consciousness. The fourth component of flow, according to [12], concerning the transformation of time was indicated by the facilitators in the form that the participants tended to continue to interact until they were more or less exhausted even after the end of the session.

My conclusion from this is that the use of the body in the interaction was motivating and created curiosity among the participants so as to continue their play and to practice their otherwise limited skills. This extends the concept of autotelic activity to also embrace the concept of aesthetic resonance. [15] refers to interactions such as reported in this paper as instrumental exploration, motivated and learned by the cause and effect and surprise exploration awaked by pure and simple novelty. This is to say that the instrumental and the surprise exploration achieved from the interactive environment are relational as forms of motivation due to the novelty [15].

\section{Spaces for Playful Learning}

This section discusses design features that influence learning in interactive environments by emphasizing open-ended interfaces and adaptation of the system through facilitator intervention. Previous research has defined the quality of intuitiveness as a crucial usability factor so as to be easy to learn and use [16]. This finding is supported by the studies presented in $[5,6,8]$, where the participants put fewer loads on cognitive processing through the immediate and aesthetically adapted feedback from the system used in the studies.

Findings presented in [17] showed that tactile, visual, and audio feedback encouraged exploration and gestures, which was identified as open-ended features of design through the alterative opportunities in the interaction that was offered through these different modalities. Affordances in the form of shapes and surfaces offered information and creative values for the interaction. The findings presented in the previous paragraph (relative to findings from $[5,6,8]$ ) add to the fact that the situation within the kinesphere [18] - augmented as a volumetric information space where the participants' gestures were mapped, translated by the interface as control data to respond with selectable multimedia content manipulation in an immediate manner - clearly offered invisible affordances for the participant to act upon. This fact was enhanced by the non-intrusive interface, which, according to [19], offers the qualities of transparency and reflectivity through the mirroring of the participant's complexity of needs and desires. Thus, I conclude that the invisible affordances of the interface exhibit a 
tactile character through the manipulation, both direct and immediate, encouraged the participant's actions within the space. This extends the definition of an open-ended interface to also embrace the invisible gestural modality of the system. These findings are relative to the findings presented in $[5,6,8]$ where the participants experienced safety and control when they interacted within the responsive environment, which empowered them to make own choices. The connection between these studies is supported by [20] 'actant' theory, where he emphasizes that other people as well as objects are equivalent as actants from the individual's perspective. The main point is that these aspects of intervention, including (i) adaptation of the system, (ii) creation of user profile, (iii) and intersubjectivity, represent different levels of practice and realization. Each of them contributes to the quality assurances based on the facilitator's judgements in and on action [21].

\section{Composition of Playful Learning and Design}

Previously in this paper it has been argued that the interactive environments used in the studies included in the paper, can be seen as a specific accessible technology allowing movement empowered by non-invasive technology. In this section an encapsulation of learning and design features within an interactive environment is presented through a model which, on the basis of the findings presented in the previous sections, describes the state and space of optimized motivation. The model which is proposed describes playful learning in interactive environments based on [12] theory on flow, Leont'ev's three level model of activity [23], and [22] theory on the Zone of Proximal Development (ZPD) in learning situations, and modified in order to better describe the processes that are dealt with here. The model was applied to better understand the findings from the individual empirical studies, but also to be able to take these findings a step further and develop a more comprehensive understanding of ludic engagement designs.

The model (fig. 1) is based on a boundary of the zone of optimization, which contains the on-going action cycles between the subject and the system as well as the continuous interventions from the outside agent to the system. These interventions can cause an ambiguity in the situation due to the fact that the goal of the action is equilibrium for a moment. The outside agent makes on-going judgements in and on the situation, i.e. when to intervene and how to adjust the multimedia feedback to fit the subject's wishes, desires, and level of competence. In this way the challenge with inherent surprises for the subject becomes optimal. The ambiguity in the situation is in the creation of an intersubjective activity, which is based on asymmetry rather than symmetric functioning. Bakhtin [25] dialogic concept of polyphony is representational of what is being approached in this situation. In other words, through the intervention more than one voice in the communication is active, the outside agent's through the intervention, the subject through the feedforward, and the system, which represents a distinct voice through the feedback. This can be thought of as a jointly created dialogue. When an increased level of shared situation takes place [26] a transfer of responsibility of the task is accomplished; i.e. the intra-subjective process. The 
ZPD is defined as the distance between the actual level of development, - which is determined through the child's own way of solving problems - and the potential level of development, - which is defined through guidance of the adult [22]. Outside the borderline is a zone representing the aesthetic resonance grow zone and the edge zone, which is relative to the ZPD [22] in that the support from the outside agent and the system as mediating tools in order for the subject to reach beyond his or her limits is emphasized; aesthetic resonance.

In this way, the state of aesthetic resonance constitutes a bridge between inter and intra-subjective processes. This also includes an intra-subjective process according to the outside agent. The outside agent is involved in multifaceted judgements and responsibilities. He or she is not only focused on achieving a specific activity, but also on the minimally required intervention (adaptation of system and/or guidance - physical or verbally) so as to best maintain the subject's interest and engagement in the situation. An understanding relative to the importance of interest, curiosity, and enjoyment are inherent as resources for learning. According to [10] the model is representational of creative processes rather than as a sequential routine as the participant within the activity situation is involved in exploring and extending their skills, intentions and ideas. Thus, my preference is to create the conditions and design the activities that most likely will lead to the desired learning I have in mind. In terms of [27] this should be focused upon the qualities as integral to the experience that leads to learning. To do this, activities that encourage actions and interaction are designed. This complexity is illustrated in Fig. 1 below.

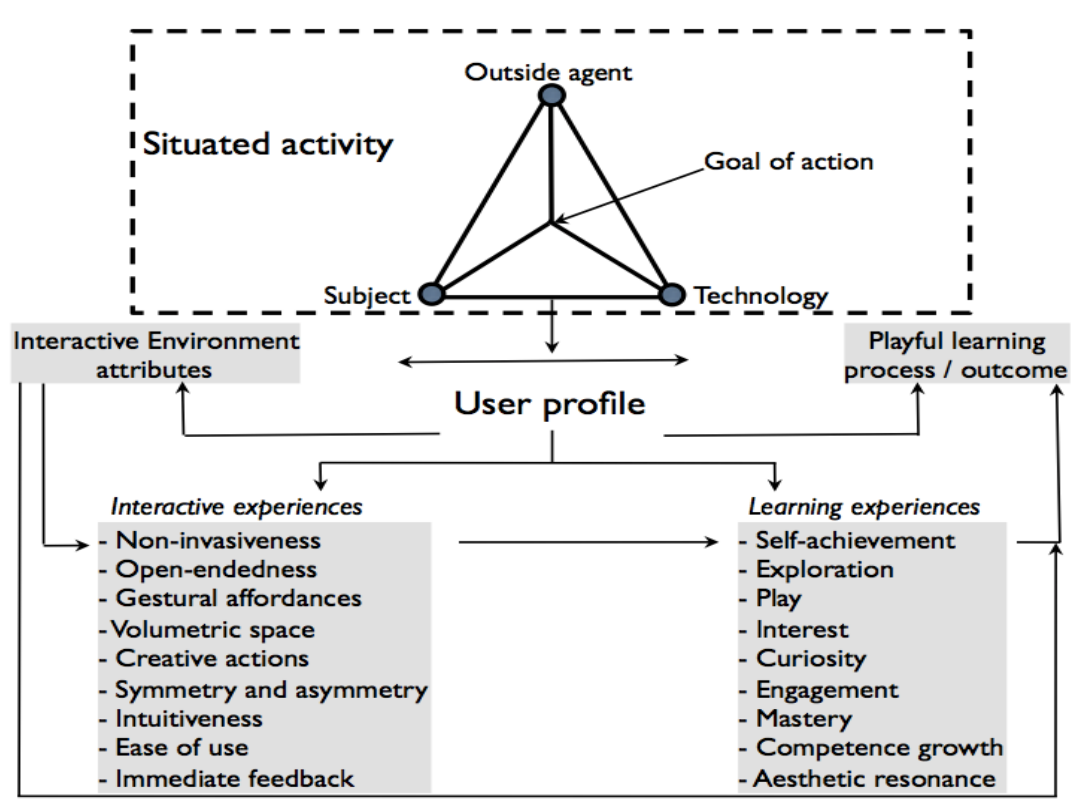

Fig. 1. The composition of learning and design in interactive environments 
The learning within and the ludic engagement design constitutes a situated activity, with inherent actions and interventions. The goal of action is established according to the user by the facilitator determining a user's initial profile, which is incrementally refined as a result of session analysis. The profile influences the facilitator's decisions on how to set up the attributes of the virtual environment relative to the desired learning process and the expected outcome of that process. These decisions influence the learning process and the outcome of that process. All in all, this is an iterative process, which supports a holistic view on the design of interactive environments towards learning.

\section{Conclusions}

The goal of this paper was to offer an approach to and definition of the concept of 'Ludic Engagement Designs'. By scrutinising affordances of interactive environments targeting learning and rehabilitation and participants' playful engagements through actions, a model was proposed describing ludic engagement designs as an approach to create spaces for playful learning. The model constitutes a foundation for the contemplation of design issues that have emerged as having a generic outcome that offers opportunities and potential for other interactive environments that have been designed as remedial play environments for children.

Multimedia feedback was synchronously manipulated from sourced data movement information from each participant. The data was sourced through invisible volumetric non-invasive sensor technology [30]. The results highlighted positive effects of the interaction within the responsive environment by those with severe disabilities as well as typically developed participants, and conclude at the potential of the concept as a supplement to traditional learning and therapy techniques. Furthermore, the results highlighted the intervention strategy with the facilitator as a key person and having a prerequisite for targeting the participant's motivated engagement and ludic experiences. The attributes of the technology and of the interactive environments supported playful learning and I reflect on the central principle of learning without expectations or rules: creating spaces that build upon and is influenced by the participant's interest. Interventions create opportunities for the facilitator to learn from his or her own involvement as they support and encourage the participants to learn.

This creation of this paper has been an act of iterative reflection and analysis. Using this approach, interpretations and understandings can be made and differences in impact of using different feedback to the user can be examined. Thus, in closing, I state that it is not possible to capture or describe other peoples' experiences, nor is it possible to understand the world exactly like it is for others. However, it is possible to strive from the perspectives of our own lives to understand how the other person experiences something and then to compare it with situations we are aware of from our own experiences. 


\section{References}

1. Petersson, E.: Editorial: Ludic Engagement Designs for All. In: Digital Creativity, vol. 19(3), pp. 141-144. Routledge, London (2008)

2. Beach, F.A.: Comparison of Copulatory Behaviour of Male Rats Raised in Isolation, Cobahitation, and Segregation. Journal of Genetic Changing World 60, 121-136 (1942)

3. Bruner, J.S.: Organization of Early Skilled Action. Child Development 44(1), 1-11 (1973)

4. Brooks, A., Petersson, E.: Recursive Reflection and Learning in Raw Data Video Analysis of Interactive 'Play' Environments for Special Needs Health Care. In: Proceedings of Healthcom 2005. 7th International Workshop on Enterprise Networking and Computing in Healthcare Industry, Busan, Korea, pp. 83-87 (2005)

5. Brooks, A., Petersson, E.: Humanics 2: Human Computer Interaction in Acquired Brain Injury Rehabilitation. In: Proceedings of HCI International 2005. Lawrence and Erlbaum Associates, Las Vegas (2005)

6. Petersson, E., Brooks, A.: Non-formal Therapy and Learning Potentials through Human Gesture Synchronized to Robotic Gesture [HRI] within a Virtual Environment [VE]. Universal Access in the Information Society 6, 166-177 (2007)

7. Wertsch, J.V.: Mind as Action. Oxford University Press, New York (1998)

8. Brooks, A., Petersson, E.: Raw emotional signalling, via expressive behaviour. In: Proceedings of the 15th International Conference on Artificial Reality and Telexistence, Christchurch, New Zealand, pp. 133-141 (2005)

9. Brooks, A., Petersson, E.: Play Therapy Utilizing the Sony EyeToy®. In: The Proceedings of Presence 2005, London, UK, pp. 303-314 (2005)

10. Tikhomirov, O.K.: The Theory of Activity Changed by Information Technology. In: Engeström, Y., Miettinen, R., Punamäki, R.-L. (eds.) Perspectives on Activity Theory. Cambridge University Press, Cambridge (1999)

11. Vygotsky, L.S.: The Genesis of Higher Mental Functions. In: Wertsch, J.V. (ed.) The Concept of Activity in Soviet Psychology. Sharpe, New York (1981)

12. Csikszentmihalyi, M.: Flow: The Psychology of Optimal Experience. Harper Perennial, New York (1991)

13. Bruner, J.S.: Nature and Uses of Immaturity. In: Bruner, J.S., Jolly, A., Sylva, K. (eds.) Play - Its Role in Development and Evolution. Basic Books, United States (1976)

14. Rogoff, B.: Apprenticeship in Thinking. Cognitive Development in Social Context. Oxford University Press, New York (1990)

15. Berlyne, D.E.: Novelty and Curiosity as Determinants of Exploratory Behaviour. Brittish Journal of Psychology 41, 61-80 (1950)

16. Bærentsen, S.: Intuitive User Interfaces. Scandinavian Journal of Information Systems 12, 29-59 (2000)

17. Petersson, E., Brooks, A.: Virtual and Physical Toys - Open-ended Features towards Nonformal Learning. Cyber Psychology and Behavior 9(2), 196-199 (2006)

18. Laban, R.: Modern Educational Dance. MacDonald and Evans, Bungay (1963)

19. Bolter, J.D., Gromala, D.: Windows and Mirrors. Interactive Design, Digital Art, and the Myth of Transparency. The MIT Press, Cambridge (2003)

20. Latour, B.: Technology is Society Made Durable. In: Law, J. (ed.) A Sociology of Monsters. Essays on Power, Technology and Domination, Routledge, New York (1991)

21. Löwgren, J., Stolterman, E.: Thoughtful Interaction Design. A Design Perspective on Information Technology. The MIT Press, Cambridge (2004)

22. Vygotsky, L.S.: Mind in Society. The Development of Higher Psychological Processes. Harvard University Press, Cambridge (1978) 
23. Leontév, A.N.: Activity, Consciousness and Personality. Prentice-Hall, Englewood Cliffs (1981)

24. Lave, J., Wenger, E.: Situated Learning. Legitimate Peripheral Participation. Cambridge University, NY (1991)

25. Bakhtin, M.M.: The Dialogical Imagination: Four Essays. In: Holquist, M. (ed.) (1994)

26. Wertsch, J.V.: Vygotsky and the Social Formation of Mind. Harvard University Press, Cambridge (1985)

27. Rogoff, B.: Becoming a Cooperative Parent in a Parent Cooperative. In: Rogoff, B., Goodman Turkanis, C., Bartlett, L. (eds.) Learning Together. Oxford University Press, New York (2001)

28. Brooks, T., Camurri, A., Canagarahah, N., Hasselblad, S.: Interaction with Shapes and Sounds as a Therapy for Special Needs and Rehabilitation. In: Proceedings of the 4th International Conference on Disability, Virtual Reality \& Associated Technologies, pp. 205212 (2002)

29. Brooks, A., Hasselblad, S.: CAREHERE - Creating Aestetically Resonant Environments for the Handicapped, Elderly and Rehabilitation: Sweden. In: Sharkey, P., McCrindle, R., Brown, D. (eds.) 5th International Conference on Disability, Virtual Reality \& Associated Technologies, pp. 191-198 (2004)

30. Brooks, A.L.: Virtual Interactive Space (V.I.S.) as a Movement Capture Interface Tool Giving Multimedia Feedback for Treatment and Analysis. In: The International Symposium for Integrative Medicine \& Expressive Therapies, pp. 3-22 (1999) 